\title{
Laser Lap Joining of Dissimilar Materials: A Review of Factors Affecting Joint Strength
}

\author{
K. F. TAMrin ${ }^{1,2}$, Y. NUKMAN ${ }^{1}$, AND S. S. ZAKARIYAH ${ }^{2,3}$ \\ ${ }^{1}$ Department of Mechanical Engineering, Faculty of Engineering, University of Malaya, \\ Kuala Lumpur, Malaysia \\ ${ }^{2}$ Advanced Technovation Ltd., Loughborough Innovation Centre, Loughborough University Science and \\ Enterprise Park (Holywell Park), Loughborough, U.K. \\ ${ }^{3}$ Photonics Research Group, Department of Electrical Engineering, Faculty of Engineering, \\ University of Malaya, Kuala Lumpur, Malaysia
}

\begin{abstract}
Laser joining of dissimilar materials has been the subject of intensive studies in the past decade or thereabout. This is mainly due to the several benefits it offers when used to manufacture various electromechanical components for automotive, aerospace, electronics, and biomedical applications. There are many possible variations of joining dissimilar materials; this article, however, considers the main engineering materials in use today, namely, metal, polymer, ceramic, glass, and silicon. The strength of the joints determined by, inter alia, the material combinations, joining technique, and material treatment is crucial for the above mentioned applications if safety and reliability requirements are to be adhered to. Undoubtedly, the challenges posed by such complex selection of materials and process factors are unquantifiable and as such have been given a critical review in this article. The relationship between some important laser processing parameters and joint strength are also discussed. Furthermore, it has been observed that the joint strength can also be influenced by factors such as bubbles morphology, material preparation/treatment, depth of molten pool and formation of chemical bonds, and intermetallic phases and their effects are also reviewed and discussed. This article is concluded with an outlook providing the summary and key findings of the authors.
\end{abstract}

Keywords Dissimilar; Joining; Joint; Laser; Material; Strength; Welding.

\section{INTRODUCTION}

Joints between dissimilar metals have received great acceptance in power generation, petrochemical, nuclear, and electronics industries [1]. Combining metals and alloys of dissimilar properties have successfully removed certain constraints and rigidity in design while paving the way for technical and economic competitiveness over singularly-fabricated components [2, 3]. Similarly, it has been established that enormous benefits can be derived by combining materials of dissimilar classes such as metal/ceramic [4-7], metal/glass [8], metal/polymer [9-18], glass/silicon [19-22], glass/polymer [23], polymer/ ceramic [24], among others.

Micro-electro-mechanical systems (MEMs) are generally fabricated on a silicon substrate due to the high adhesive property of the latter to a variety of materials [20]. Due to this outstanding mechanical strength, silicon-based microsystems are used for sensing and actuating purposes [19]. However, silicon has inferior resistivity to moisture [25] and is therefore generally replaced with polymer to overcome this problem. Meanwhile, thermoplastic polymers such as polyimide (PI)

Received November 3, 2012; Accepted March 4, 2013

Address correspondence to K. F. Tamrin, Department of Engineering Design and Manufacture, Faculty of Engineering, University of Malaya, 50603 Kuala Lumpur, Malaysia; E-mail: krolfikri @yahoo.co.uk
$[9,10,14,15,17,26]$, polyetheretherketone (PEEK) [27], polyethylene terephthalate (PET), [28-32], and parylene [25] show good resistance against some chemicals and thermal degradation. In particular, parylene has inert characteristics, which makes it a more suitable and useful material [25]. Thermoset polymers cannot be welded without additional intermediate layer; this is because their molecules cannot be reheated or re-formed [33]. Joints between dissimilar materials classes in MEMs and Bio-MEMs applications must fulfill tough requirements in terms of strength [23], thermal stress, fatigue, hermeticity [34], and long term stability [27, 35]. As a result, polymeric materials are usually combined with metals to fulfill the aforementioned test requirements. Commonly used metals for this application include titanium [9-18], kovar (nickel-cobalt ferrous alloy) [4, 36], platinum, gold, and stainless steel $[28,29]$. Titanium and nitinol are some of the biocompatible metals being used for medical implants. Nitinol-also known as a "shape memory alloy" (SMA) because of its ability to "remember" and regain its original shape after undergoing deformation - is a suitable choice for applications in aerospace and medical sectors [27].

High demand for lightweight components to reduce cost, weight, and increase productivity has led to numerous designs of hybrid components and structures [3740]. For example, carbon fiber composites have been widely used in aircraft and automotive constructions as they offer weight reduction while retaining good 